Pulsars : Problems \& Progress

ASP Conference Series, Vol. 105, 1996

S. Johnston, M. A. Walker and M. Bailes, eds.

\title{
Monitoring Millisecond Pulsars in Full Polarization
}

\author{
Kiriaki M. Xilouris \\ National Astronomy and Ionospheric Center-Arecibo Observatory, P.O. \\ Box 995, Arecibo, PR 00613 \\ Michael Kramer \\ Max-Planck-Institut für Radioastronomie, Auf dem Hügel 69, 53121, \\ Bonn, Germany
}

\begin{abstract}
Over a two year period the polarization properties of the largest sample of millisecond pulsars yet observed have been regularly monitored with the Effelsberg $100-\mathrm{m}$ radiotelescope at $\lambda 21 \mathrm{~cm}$. A comparison of the observed emission properties with those of the population of long period pulsars is presented here. Most of the sources exhibit wide, often multiple-component profiles, with complex polarization features, not easily interpretable in the context of the well established phenomenological models used to describe the properties of long period pulsars. While both groups share common emission features suggesting similar emission processes, the implied field structures as revealed from polarization information appear substantially different from a dipolar structure. Generally, short period pulsars exhibit a relatively large percentage of circularly polarized emission while at $\lambda 21 \mathrm{~cm}$ they tend to be less polarized than long period pulsars.
\end{abstract}

\section{Introduction}

The great diversity in pulsar emission properties has restricted the processes which can be active in pulsar magnetospheres. Current modeling, though mostly phenomenological in nature, depends primarily on trends recognized in the emission properties. Among these are the average profile width and its frequency dependence, intrinsic intensity fluctuations and polarization. While considerable information exists for the population of long period pulsars, similar studies for the millisecond pulsars are scarce (e.g. Thorsett \& Stinebring, 1990). Recently, we observed a sample of 21 millisecond pulsars (Xilouris et al. in prep.) at $\lambda 21$ $\mathrm{cm}$ using a $60 \times 0.66 \mathrm{MHz}$ filter bank with the Effelsberg $100-\mathrm{m}$ radiotelescope. The complexity seen in the mean profiles and the perplexing polarization features motivated a comparison between millisecond pulsar emission properties and those of long period pulsars. In this contribution the results are used to probe the implications of the thousand fold faster rotation on radioemission. 


\section{Comparison of Emission Properties}

It has long been known (see e.g. Kramer et al. 1994) that the mean pulse profiles of long period pulsars can be described by a superposition of several $(\lesssim 5)$ Gaussian components. In this way the structure of the active magnetospheric regions can be derived, a phenomenological concept, that has proved useful in deriving the geometry and classifying long period pulsars (e.g. Rankin 1983). In Fig. 1, we present the distribution of half-power profile widths as a function

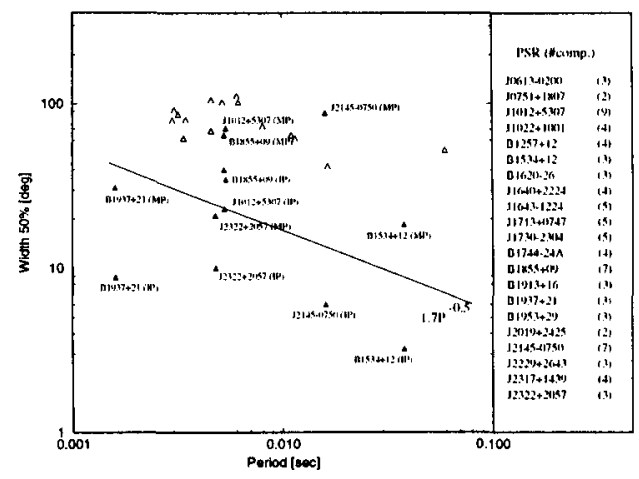

Figure 1. Half-power widths of millisecond pulsar mean profiles as a function of period.

of period for 21 millisecond pulsars. The widths presented are derived from the Gaussian fits to the mean pulse profiles using the procedure developed by Kramer et al. (1994). In the column included in Fig. 1, the sources accompanied by the number of fitted components are given. For long period pulsars, this distribution has a relatively well defined lower boundary, (here given as W [deg] $=1.7 \cdot \mathrm{P}[\mathrm{s}]^{-0.5}$, see Gould 1994). The actual functional dependence on period, which has also significant implications for the dependence of emission altitudes, has been the subject of both theoretical and observational controversy. Much hope to clarify this issue was placed on studies of millisecond pulsar profiles, in particular if a clear distinction between single pole and double pole emission is possible. In such a case one can decide whether a measured width represents a true cut of the emission beam (and thus represents a reasonable estimate about its diameter) or whether it corresponds to a less representative grazing along the beam. The complexity seen in polarization data did not allow for such a distinction since the phenomenology applied to long period pulsars is not directly evident in the msec-pulsar profiles. In Fig. 1 we use open triangles to mark pulsars considered to exhibit emission from a single pole, while filled triangles (individually identified) refer to main pulses (MPs) and interpulses (IPs) of more complex profiles. We note that PSRs B1937+21, B1534+12 and J2322+2057, which represent the most clear cases of double pole emission, exhibit main pulses and interpulses much narrower than the majority of the other studied sources. In contrast, more complex cases, i.e. PSRs J1012+5307, B1855+09 or J2145-0750, have components mostly above the shown boundary line. This observation is interesting since the boundary line was derived from long period pulsars (Gould 
1994), where a dipolar field structure persists in the emission region (Kramer \& Xilouris, these proceedings). In such a case, the ratio of emission altitude and light cylinder radius is then independent of pulse period. If short period pulsars follow a similar trend, their active magnetospheric regions would therefore correspond to an appropriate scaled version of those of long period pulsars. However, if some cases resembling a MP-IP structure exhibit much narrower components than expected from the boundary line, it might give support to a migration of magnetic poles as suggested by Chen \& Ruderman (1993). The number of components shown in Fig. 1, ranges from 2 to 9 , while simpler structures such as single or double profiles are rare.
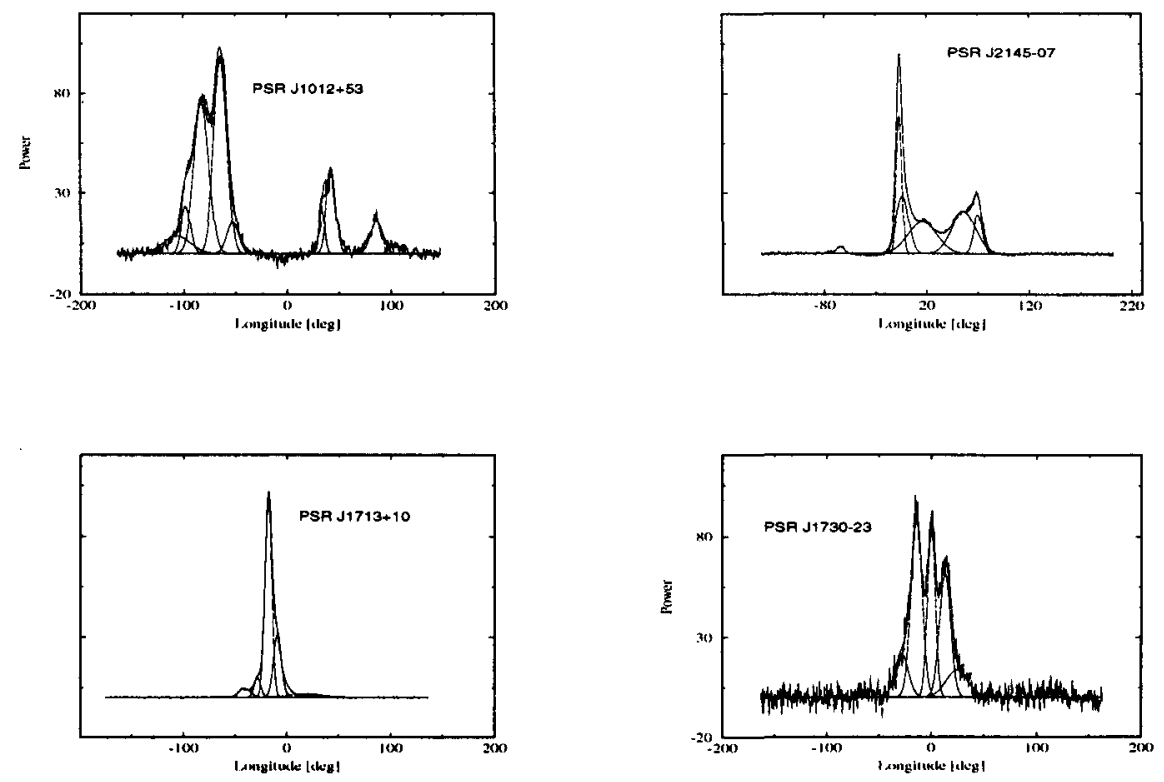

Figure 2. Mean Pulse profiles for multicomponent millisecond pulsars with the Gaussian components overplotted on the data

Examples of profiles with the fitted components superimposed are presented in Fig. 2. PSR J1012+5307 resembles closely a much long period pulsar, B105552 (e.g. Lyne \& Manchester, 1988). While other examples can be found within the long period pulsar population, resembling the complexity seen in millisecond pulsars, it should be mentioned that they are very rare and they are mainly among the wide profiles which are thought to represent aligned geometries. However, the opening angles of long period pulsar components are much narrower, typically a few degrees, in comparison to the more than 10 degrees often measured in the majority of short period pulsars. This also leads to the generally wider emission beams of millisecond pulsars, which are now finally evident by Fig. 1 and expected due to the smaller light cylinder radii of short period pulsars.

Low-level linear polarization is the dominant feature of the $\lambda 21 \mathrm{~cm}$ polarization profiles (Fig. 3). Usually, in long period pulsars at this frequency the emission is highly polarized, while in the case of millisecond pulsars the trend 
is towards depolarization. Minima in the linear polarization curves, associated with $90^{\circ}$ jumps in position angle are frequent features, a well-established characteristic of the emission in long period pulsars. Circular polarization, usually of alternating sense, is present in many cases. This is a known, but relatively rare, feature of long period pulsars, particularly at such high percentages as seen here. The polarization position angle is often disturbed by orthogonal modes and in
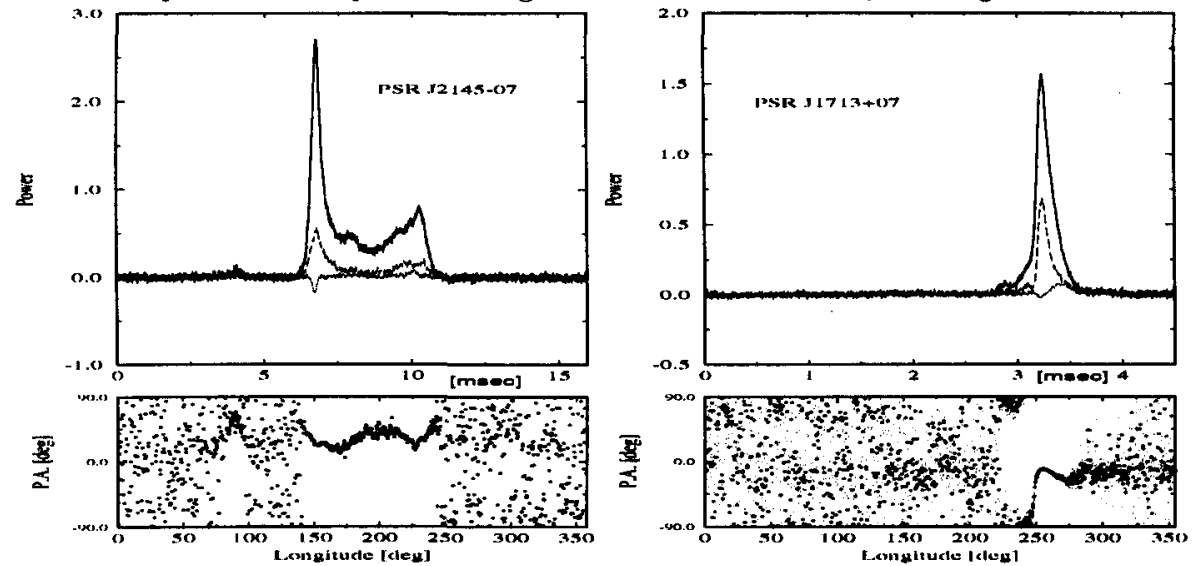

Figure 3. Millisecond pulsar polarization profiles. Total power, linear polarization and circular polarization are plotted in the upper pannels, while the curve in the lower pannels represents the linear polarization position angle across the profile.

some cases it can be reconstructed to resemble a dipolar field geometry. In other more difficult cases (e.g. PSR J2145-0750), where there is also complex emission throughout a significant fraction of the rotational period, such a reconstruction is not easily achieved. In fact, the polarization position angle appears highly disturbed, a feature often associated with the presence of higher older magnetic field moments.

Acknowledgments. We would like to thank O. Doroshenko, A.v. Hoensbroech, A. Jessner and J. Kijak for their help with the observations. We are also very grateful to D.R. Lorimer for his helpful comments. Arecibo Observatory is operated by Cornell University, under a cooperative agreement with the National Science Foundation.

\section{References}

Gould, D.M. 1994, Ph.D thesis, The University of Manchester

Kramer, M., Wielebinski, R., Jessner, A., Gil, J.A., Seiradakis, J.H. 1994, A\&A, 107,515

Lyne, A. \& Manchester, R. 1988, MNRAS, 234, 477

Chen, K. \& Ruderman, M. 1993, ApJ, 408, 179

Rankin, J.M. 1983, ApJ, 274, 333

Thorsett, S. E. \& Stinebring, D. 1990, ApJ, 361, 644 